\title{
Submicron YBCO Josephson junctions on sapphire bicrystal substrates for microwave devices
}

\author{
E. Stepantsov ${ }^{\text {a,b,* }}$, M. Tarasov ${ }^{\text {a,c }}$, A. Kalabukhov ${ }^{\text {a,d }}$, T. Lindström ${ }^{\text {a }}$, \\ Z. Ivanov ${ }^{\mathrm{a}}$, T. Claeson ${ }^{\mathrm{a}}$ \\ a MINA, Chalmers University of Technology and Göteborg University, Göteborg, SE-41296, Sweden \\ ${ }^{\mathrm{b}}$ Institute of Crystallography, Leninsky Prosp. 59, Moscow 117333, Russia \\ ${ }^{\mathrm{c}}$ Institute of Radio Engineering and Electronics, Mokhovaya 11, Moscow 101999, Russia \\ d Department of Physics, Moscow State University, 119899 Moscow, Russia
}

\begin{abstract}
Submicron YBCO bicrystal Josephson junctions (JJ) and devices for high frequency applications were designed, fabricated and experimentally studied. The key elements of these devices are bicrystal sapphire substrates with symmetric misorientation angles $14.2^{\circ}, 18.4^{\circ}$ and $24^{\circ}$ which were fabricated by a solid-state intergrowth method. A technological process based on deep ultraviolet photolithography using a hard carbon mask was developed for the fabrication of $0.4-0.6 \mu \mathrm{m}$ wide JJ. The junctions have normal resistances up to $2 \mathrm{k} \Omega, I_{\mathrm{c}} R_{\mathrm{n}}>2 \mathrm{mV}$ and a $1 \mathrm{mV} / \Phi_{0}$ SQUID response at $4.2 \mathrm{~K}$. The junctions were used as Josephson detectors and spectrometers at frequencies up to 1.5 $\mathrm{THz}$, dc SQUID RF amplifiers operating at $1.5 \mathrm{GHz}$ and submillimeter wave array oscillators.
\end{abstract}

(c) 2002 Elsevier Science B.V. All rights reserved.

Keywords: High critical temperature superconductors; Sapphire bicrystal substrates; Josephson junctions; Microwave sensors

\section{Introduction}

Terahertz applications of Josephson junctions (JJ) have gained much attention lately. For optimal operation of Josephson microwave detectors (see Ref. [1] for theory) the characteristic voltage should be above the normalised frequency $V_{\mathrm{c}}>$ $f \Phi_{0}$, where $V_{\mathrm{c}}=I_{\mathrm{c}} R_{\mathrm{n}}$ is the characteristic voltage, $I_{\mathrm{c}}$ the critical current, $R_{\mathrm{n}}$ the junction normal re-

\footnotetext{
* Corresponding author. Address: MINA, Chalmers University of Technology and Göteborg University, Göteborg, SE41296, Sweden. Tel.: +46-031-7723117.

E-mail address: evgueni@fy.chalmers.se (E. Stepantsov).
}

sistance, $f$ the signal frequency, and $\Phi_{0}=h / 2 e=$ $2 \times 10^{-15} \mathrm{Tm}^{2}$ is a flux quantum. The $V_{\mathrm{c}}$ values of the order of 2-4 $\mathrm{mV}$ have been achieved in high temperature superconductor $\mathrm{JJ}$ of different type e.g. bicrystal, step edge or ramp junctions. It should be noted that in most cases the substrates were $\mathrm{SrTiO}_{3}$ or $(\mathrm{Y}) \mathrm{ZrO}_{2}$ with high microwave losses. Sapphire substrates are attractive for $\mathrm{THz}$ devices because of the low losses and low dielectric constant. A number of papers have addressed JJ on sapphire bicrystal substrates with high misorientation angles [2-4]. Here we report on the development of a technology for submicron $\mathbf{J} \mathbf{J}$ on low angle sapphire bicrystal substrate. Junctions integrated with log-periodic antennas were tested 
in the frequency range 63-500 GHz. Junctions parameters were also evaluated for SQUID amplifiers.

\section{Fabrication}

Sapphire bicrystals are formed by solid-state intergrowth of two monocrystals oriented so that the plane of contact (grain boundary plane) is orthogonal to the $(10 \underline{12})$ planes of the crystals and makes a $33^{\circ}$ angle with their (1 120$)$ planes. The bulk bicrystal was diced along the common (1 0112 ) plane. The final stage included a chemicalmechanical polishing of the bicrystals and dicing them into $5 \times 5 \mathrm{~mm}^{2}$ substrates with the grain boundary in the centre. The $\mathrm{CeO}_{2}$ and $\mathrm{YBa}_{2} \mathrm{Cu}_{3}-$ $\mathrm{O}_{7-\delta}(\mathrm{YBCO})$ films were grown by pulse deposition with a $\mathrm{KrF}$ excimer laser at a pulse rate of 10 $\mathrm{Hz}$. The distance between target and substrate was $50 \mathrm{~mm}$ in all cases. A thin $\mathrm{CeO}_{2}$ buffer layer was initially deposited to prevent the chemical interaction of the YBCO with sapphire. To minimize the $\mathrm{YBCO} / \mathrm{CeO}_{2}$ interaction a $20 \mathrm{~nm} \mathrm{YBCO}$ film was initially grown at reduced temperature, 750 ${ }^{\circ} \mathrm{C}$, which was followed by deposition of $250 \mathrm{~nm}$ YBCO film at $790{ }^{\circ} \mathrm{C}$. Deposition parameters for all layers are listed in Table 1. Finally, a $20 \mathrm{~nm}$ gold thin film was deposited in situ at $100{ }^{\circ} \mathrm{C}$. Atomic force microscope (AFM) images show that the films are free of $a$-axis particles and the density of outgrowths, presumably $\mathrm{CuO}$ and $\mathrm{Y}_{2} \mathrm{O}_{3}$ does not exceed 1 per $200 \mu^{2}$. The critical temperature of the films was above $89 \mathrm{~K}$.

Microbridges with width varying between 300 $\mathrm{nm}$ and $1.5 \mu \mathrm{m}$ were pattern across the grain boundary to form JJ. A deep ultraviolet lithogra- phy, with $\lambda=248 \mathrm{~nm}$, was used to define submicron patterns in the Microposit UV-5 photoresist, that was later transferred into a three layer hard mask, consisting of $\mathrm{Au}(50 \mathrm{~nm}) / \mathrm{a}$-carbon $(100 \mathrm{~nm}) /$ $\mathrm{Au}(50 \mathrm{~nm})$. Through the resist pattern the top layer of gold was etched by $\mathrm{Ar}^{+}$ion beam with an energy of $250 \mathrm{eV}$ and current density of $0.2 \mathrm{~mA} /$ $\mathrm{cm}^{2}$, afterwards the residual resist and carbon were removed through the opened areas in an oxygen plasma. The required pattern was formed in the YBCO film by using $\mathrm{Ar}^{+}$beam etching through the carbon mask. The rest of the carbon was removed in oxygen plasma ( $5 \mathrm{~min}, 250 \mathrm{~W}, 10 \mathrm{mbar}$ ). Finally, the $20 \mathrm{~nm}$ in situ gold layer was removed by $\mathrm{Ar}^{+}$ beam etching. The described technique allows us to prepare bicrystal bridge junctions crossing the grain boundary that are aligned with an accuracy of about $2 \mu \mathrm{m}$ (see Fig. 1). The typical bridge has a

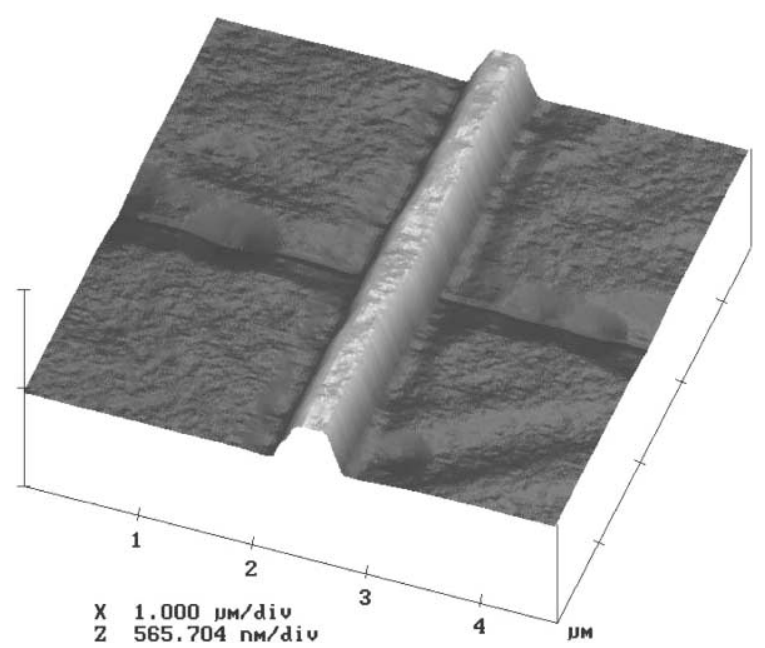

Fig. 1. Submicron YBCO JJ at the bicrystal boundary. The width at the top is $0.4 \mu \mathrm{m}$ and at the bottom $0.6 \mu \mathrm{m}$.

Table 1

Process parameters for pulsed laser deposition

\begin{tabular}{lclllll}
\hline Layer & Thickness $(\mathrm{nm})$ & $\begin{array}{l}\text { Deposition rate } \\
(\mathrm{nm} / \mathrm{min})\end{array}$ & $\begin{array}{l}\text { Oxygen pressure } \\
(\mathrm{mbar})\end{array}$ & $\begin{array}{l}\text { Substrate temper- } \\
\text { ature }\left({ }^{\circ} \mathrm{C}\right)\end{array}$ & $\begin{array}{l}\text { Pulse energy den- } \\
\text { sity }\left(\mathrm{J} / \mathrm{cm}^{2}\right)\end{array}$ & $\begin{array}{l}\text { Pulse energy } \\
(\mathrm{mJ})\end{array}$ \\
\hline $\mathrm{CeO}_{2}$ & 15 & 20 & 0.15 & 770 & 1.1 & 55 \\
$\mathrm{YBCO}$ & 20 & 35 & 0.6 & 750 & 1.9 & 95 \\
$\mathrm{YBCO}$ & 250 & 35 & 0.6 & 790 & 1.9 & 95 \\
$\mathrm{Au}$ & 50 & 18 & - & 20 & $\begin{array}{l}\text { Thermal } \\
\text { evaporation }\end{array}$ \\
\hline
\end{tabular}




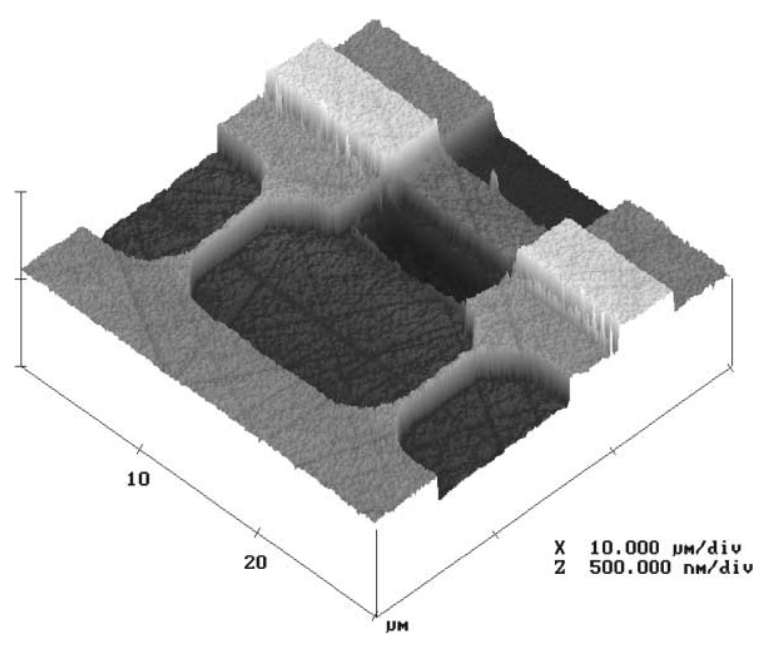

Fig. 2. A dc SQUID on a sapphire bicrystal substrate. The junction width is less than $0.5 \mu \mathrm{m}$.

trapezoidal shape with a $0.6 \mu \mathrm{m}$ bottom and $0.4 \mu \mathrm{m}$ top width. The junctions were integrated in planar log-periodic antennas and SQUID loops (Fig. 2) in order to investigate their properties.

\section{Measurements}

JJ on bicrystal substrates with misorientation angles of $24^{\circ}, 18.4^{\circ}$ and $14.2^{\circ}$, were studied. The

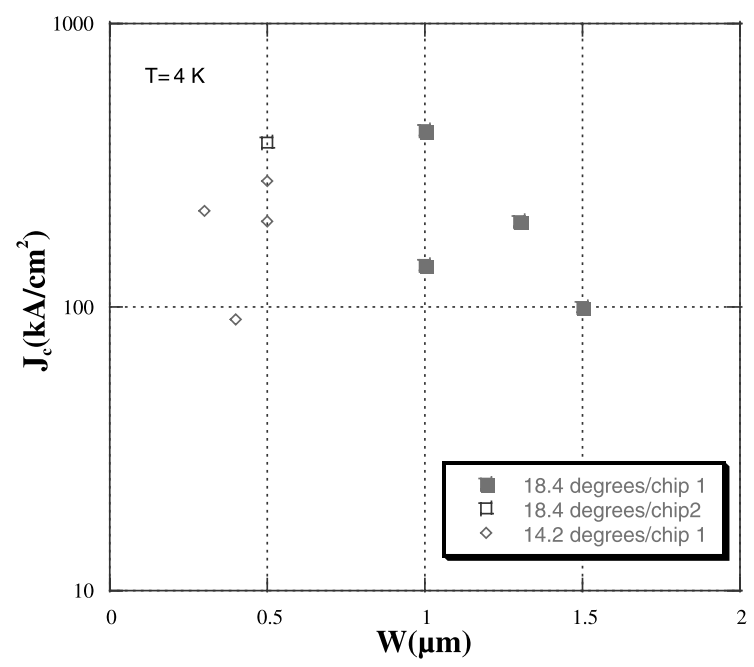

Fig. 3. Critical current vs. junction width for several submicron junctions.

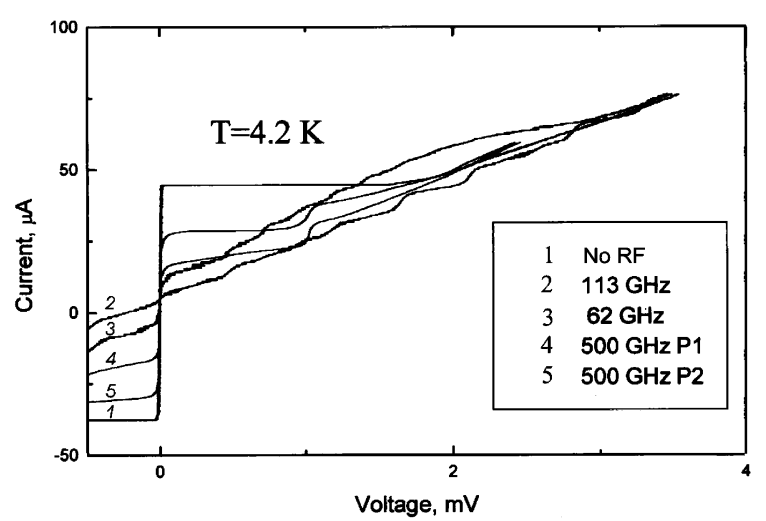

Fig. 4. $I-V$ curves of a Josephson receiving structure under irradiation. The frequency is 113,62 and $500 \mathrm{GHz}$, the later at two different levels of power.

current-voltage characteristics can be well described by the resistively shunted junction model. It is worth noting that all submicron junctions had almost no excess current. The junctions have normal resistances up to $2 \mathrm{k} \Omega$ and characteristic voltages over $2 \mathrm{mV}$. We do see a decrease of the critical current density for submicron junctions (Fig. 3) that can be explained by damage during processing or by the faceting of the grain boundary. It should however be noted that even $300 \mathrm{~nm}$ wide junctions had a $J_{\mathrm{c}}$ of $10^{4} \mathrm{~A} / \mathrm{cm}^{2}$.

Junctions integrated with log-periodic antennas were tested in the frequency range $63-500 \mathrm{GHz}$. Well pronounced Shapiro steps were observed in the $I-V$ curve at all frequencies (Fig. 4). Similar junctions and arrays have also been used for

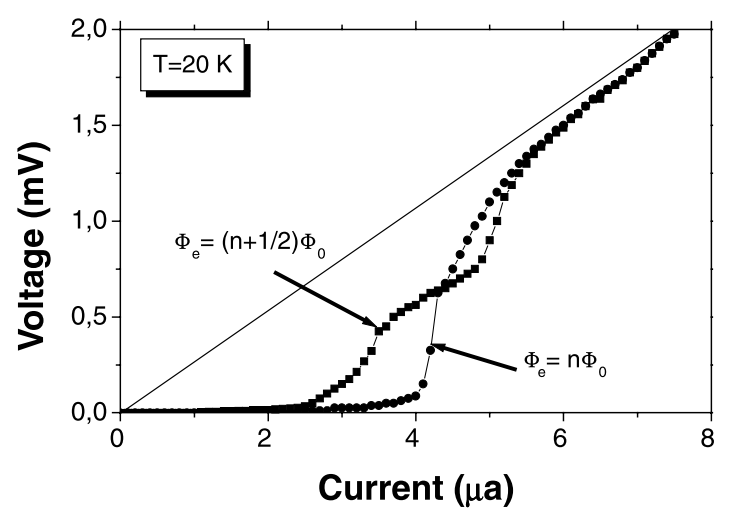

Fig. 5. $I-V$ curves from a de SQUID at two levels of external magnetic flux. 
Hilbert spectroscopy [5,6]. Parallel arrays of such junctions can also be used as electrically tuned oscillators [7].

SQUIDs with $0.5 \mu \mathrm{m}$ wide bicrystal junctions were fabricated and tested. A voltage response as high as $1 \mathrm{mV}$ was measured at $4.2 \mathrm{~K}$. (Fig. 5). These SQUIDs were also tested as RF amplifiers at a signal frequency of $1.5 \mathrm{GHz}$ [8].

\section{Conclusion}

High-quality YBCO JJ have been fabricated on sapphire bicrystal substrates. By using sapphire as a substrate material and making submicrometer size junctions the required parameters for microwave devices were achieved: a characteristic voltage over $2 \mathrm{mV}$, a SQUID voltage response over $1 \mathrm{mV}$ and a normal resistance up to $2 \mathrm{k} \Omega$. These parameters together with very low losses at RF and submillimeter waves in sapphire substrate make such junctions perfect candidates for high frequency applications e.g. Josephson detectors and spectrometers, SQUID amplifiers and oscillators.

\section{Acknowledgements}

The authors acknowledge the financial support from the Swedish Royal Academy of sciences, the Swedish Institute, EU-INTA-01-686, ISTC and the Swedish OXIDE program.

\section{References}

[1] K. Likharev, Dynamics of Josephson junctions and circuits, Gordon and Breach Science Publishers, New York, 1986.

[2] D.E. Oates et al., IEEE Trans. Appl. Supercond. 9 (1999) 2446.

[3] L.R. Vale et al., IEEE Trans. Appl. Supercond. 7 (1997) 3193.

[4] G. Kunkel et al., IEEE Trans. Appl. Supercond. 7 (1997) 3339.

[5] M. Tarasov et al., Supercond. Sci. Technol. 12 (1999) 995-997.

[6] M. Tarasov et al., Proceeding of EUCAS-1999, Applied Superconductivity 1999, Vol. 2, Institute of Physics Conference Series 167, 1999, pp. 611-614.

[7] M. Tarasov et al., Physica C; these Proceedings.

[8] M. Tarasov et al., Proc. 85th Int. Supercond. Electronics Conf., June 19-22, 2001 Osaka, Japan, pp. 149-150. 